\section{Hot solution}

SIR-Nelson's report (Nature 344, $115-116 ; 1990)$ that cables and instrumentation in remote locations can be protected by the application of Tabasco sauce and silicone sealant is a reminder that those who tend orchards also have problems with masticatory and/or curious animals, and that there is a product which is much more potent, easier to apply and (if bought in bulk) cheaper than Tabasco. This is Hot Sauce Animal Repellent (Miller Chemical and Fertilizer Corp., Hanover, Pennsylvania), which is a 2.5 per cent solution of capsaicin extracted from capsicum peppers. I have found this to be effective in protecting young almond trees, although I have heard anecdotal reports of deer that have apparently acquired a taste for this spicy fare. But Nelson's addition of silicone sealant seems to me to be an improvement.

Daniel B. HRDY

Division of Infectious Diseases

University of California Davis Medical

Center,

Sacramento, California 95817, USA

\title{
Methods of leprosy prevention
}

SIR-Bloom does not discuss the use of resistance to dapsone. leprosy vaccines in his commentary article " Vaccines for the Third World". Is this because vaccines are not the right approach to the eradication of leprosy? The simple, practical, alternative measure is to treat the patients with dapsone, kill the bacteria and prevent the spread of the disease. Where this has been done and measurements carried out there has been a dramatic decline in the disease, for example, in northern Zaria state, northern Nigeria, the prevalence of leprosy fell from 46 per 1,000 in 1953 to 1.6 per 1,000 in 1967. In the village of Igabi it fell over the same period from 67 per 1,000 to 2 per 1,000 (ref. 2).

I have previously presented ${ }^{3}$ evidence for a decline in incidence. Since then, dapsone resistance has increased but has not caused a resurgence of the disease at least in Katsina Province, northern Nigeria, where the prevalence in 1987 was 2 per 1,000 down from 39 per 1,000 in 1951 (ref. 4). The introduction of multi-drug therapy may lessen the frequency of

\section{What are sisters good for?}

SIR-An important and prevalent error surfaced in a recent Scientific Correspondence $^{1}$, in which Conover questioned why female/female pairings among birds are so rare, given that females in such pairs generally produce fertile eggs. Conover correctly pointed out that pairs of unrelated females would have to produce twice as many offspring as male/female pairs to attain the same average reproductive success per female. His mistake was to assert that sister/sister pairs must produce only 33 per cent more young than male/female pairs. This would be true only under very improbable circumstances, and then only for one of the two sisters.

Suppose, for example, that female/ female pairs fledge four young (two per female) and male/female pairs fledge three young, on average per breeding season. Thus, if homosexually paired females are unrelated, each gets credit for two young fledged. If the females are sisters, then according to Conover, their average inclusive fitness should equal that of females paired heterosexually (three young, in this example). This is the result one obtains by applying the erroneous "simple weighted sum" definition of inclusive fitness ${ }^{2.3}$ in which individuals are credited with all of their own offspring plus all of their relative's offspring (devalued by relatedness).

Hamilton ${ }^{4}$ defined inclusive fitness as that portion of individual fitness not attributable to the positive or negative influences of others, plus any harms or benefits conferred on others (weighted by rela-

tedncss). The appropriate question is whether a female pairing with her sister benefits her sister sufficiently to offset the cost of not pairing with a male, $(b \geqslant$ $c / r)$. If both sisters could produce the same number of offspring by pairing with an unrelated female, then there would be no benefit to pairing with a sister. In fact, each would suffer an inclusive fitness decrement due to the cost her sister would incur by not pairing with a male. Only in extremely asymmetrical relationships would one of the sisters in a homosexual pair get credit for as many genetic replicates as a female in a male/female pair (again, using the above hypothetical numbers of fledglings). Specifically, if a given female could fledge a total of four young with either an unrelated female or her sister, but her sister's only reproductive opportunity were to pair with her, then her inclusive fitness would equal three young if she paired with her sister versus two young if she paired with an unrelated female. Although theoretically possible, the situation is not likely to occur in nature.

Animal Behaviour Group,

GREGORYF. GRETHER ANNALISA M. WEAVER

Department of Anthropology,

University of California,

Davis,

California 95616, USA

\footnotetext{
1. Conover, M.R. Nature 342, 624-625 (1989)

2. Graten. A. Nature $298,425-426$ (1982)

3. Graten. A in Behavioural Ecology: An Evolutionan Approach 2 nd edn (eds Krebs. J.R. \& Davies. N. B.) 62-84 (Blackwell, Oxfo:d, 1984).

4. Hamilton. W.D. J. theor. Biol. 7. 1-52 (1964)
}

There are still areas where leprosy is endemic and where patients do not have access to this therapy. Even in those areas where it is available the decline in incidence is rarely recorded. In my opinion, failure to extend this treatment and ensure proper evaluation while trying to develop an effective vaccine is irresponsible, especially as the presence of the antibody against the human immunodeficiency virus may make people more vulnerable to leprosys.

Department of Anatomy,

C. L. CRAWFord

Charing Cross and Westminster

Medical School,

University of London,

London W6 8RF, UK

BLOOM REPLIES-Although dapsone therapy has made a significant contribution to the treatment of leprosy in the past, WHO has recommended multidrug therapy with three antibiotics (including dapsone) for several reasons. Multidrug therapy is more effective, has thus far blocked the emergence of drug-resistant organisms that is one of the main problems with single drug treatment, prevents relapses after treatment and can reduce the duration of treatment from a lifetime to a few years or perhaps less. Serious efforts are being made by WHO, the International Leprosy Organization and many endemic countries to extend multidrug therapy to as many patients as possible. Just under half of registered patients worldwide are now receiving this type of treatment. Finally, because of the long latency of the infection, transmission of leprosy frequently occurs before treatment can begin.

The point of developing a vaccine is to prevent infection and disease. It was an omission in my commentary not to mention that four candidate anti-leprosy vaccines are currently in clinical trials.

BARRY R. BLOON

\section{Department of Microbiology and}

Immunology,

Albert Einstein College of Medicine,

Bronx,

New York 10461, USA

1. Bloom, B.R. Nature 342, 115-120 (1989).

2. Crawford. C.L. Lepr. Rev. 40, 159-163 (1969)

3. Crawtord. C.L. Nature 254, 168-170 (1975)

4. Waaldijk, K. Lepr Rev. 60,59-61 (1989)

5. Meerar. K. Br. med. J. 264, 364-365 (1989)

\section{Scientific Correspondence}

Scientific Correspondence is intended to provide a forum in which readers may raise points of a scientific character. They need not arise out of anything published in Nature. In any case, priority will be given to letters of fewer than 500 words and five references. 